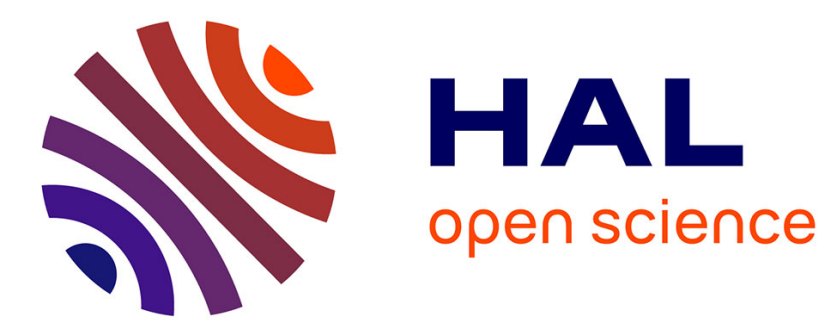

\title{
New (1-Phosphanylferrocen-1'- and -2-yl)methyl-Linked Diaminocarbene Ligands: Synthesis and Rhodium(I) Complexes
}

\author{
Agnès Labande, Jean-Claude Daran, Eric Manoury, Rinaldo Poli
}

\section{- To cite this version:}

Agnès Labande, Jean-Claude Daran, Eric Manoury, Rinaldo Poli. New (1-Phosphanylferrocen-1'- and -2-yl)methyl-Linked Diaminocarbene Ligands: Synthesis and Rhodium(I) Complexes. European Journal of Inorganic Chemistry, 2007, 2007 (9), pp.1205-1209. 10.1002/ejic.200601193 . hal-03194610

\author{
HAL Id: hal-03194610 \\ https://hal.science/hal-03194610
}

Submitted on 9 Apr 2021

HAL is a multi-disciplinary open access archive for the deposit and dissemination of scientific research documents, whether they are published or not. The documents may come from teaching and research institutions in France or abroad, or from public or private research centers.
L'archive ouverte pluridisciplinaire HAL, est destinée au dépôt et à la diffusion de documents scientifiques de niveau recherche, publiés ou non, émanant des établissements d'enseignement et de recherche français ou étrangers, des laboratoires publics ou privés. 


\section{New (1-phosphanylferrocen-1'- and 2-yl)methyl-Linked Diaminocarbene Ligands: Synthesis and $\operatorname{Rh}(\mathrm{I})$ Complexes}

Agnès Labande*, Jean-Claude Daran, Eric Manoury and Rinaldo Poli*

Laboratoire de Chimie de Coordination, UPR CNRS 8241 (lié par convention à l'Université Paul Sabatier et à l'Institut National Polytechnique de Toulouse), 205 route de Narbonne, 31077

Toulouse Cedex 4, France. E-mail : agnes.labande@lcc-toulouse.fr

Keywords: Carbene ligands / Phosphane ligands / Ferrocenes / Rhodium

Abstract: Two ferrocenyl alcohols, (1'-diphenylthiophosphinoferrocen-1-yl)methanol $\mathbf{3}$ and (2diphenylthiophosphinoferrocen-1-yl)methanol 6, have been converted in one step into the 1,1'- and 1,2- thiophosphine/N-R-imidazolium salts 4a,b and 7a,b (a: $\mathrm{R}=\mathrm{Me} ; \mathbf{b} \mathrm{R}=2,4,6-\mathrm{Me}_{3} \mathrm{C}_{6} \mathrm{H}_{2}$ or $\mathrm{Mes}$ ) This straightforward method allows the linkage of an imidazolium group to a ferrocene with a nonsubstituted methylene bridge. After desulfurisation of the phosphine, the ligands reacted with a $\mathrm{Rh}(\mathrm{I})$ precursor, in the presence of $t$-BuOK, to give cationic complexes 9a,b and 10a,b. All compounds have been characterised by elemental analysis, NMR spectroscopy and mass spectrometry. The molecular structures of compounds 4a, 7a and 9a have been determined by X-ray crystallography.

The chemistry of $\mathrm{N}$-Heterocyclic carbenes (NHCs) has experienced a very rapid development over the past ten years. ${ }^{[1]}$ They have found many applications in catalysis, since the corresponding transition metal complexes have proven to be very active, robust and generally air stable. ${ }^{[2]}$ Ligands associating a NHC and a phosphine have also shown very interesting properties, ${ }^{[3]}$ in particular for C-C coupling reactions catalysed by palladium or nickel. ${ }^{[4]}$

To date, there are only two synthetic methods allowing the preparation of ferrocenyl phosphineNHC ligands with an aryl (or ferrocenyl) substituent on the imidazole moiety. ${ }^{[5],[6]}$ However, the carbon situated in $\alpha$ to the imidazolium has a methyl substituent in both cases. To our knowledge, there is no precedent in the literature for 1,1'-disubstituted ferrocenyl phosphine-imidazolium ligands. On the other hand, the fundamental difference between our 1,2-disubstituted ligands and previously reported ligands is that the former possess only planar chirality, whereas the latter have both planar and central chirality. ${ }^{[5],[6]}$ Thus it becomes possible to study the specific effect of planar chirality in asymmetric catalytic reactions. We present here a new synthetic method for 1,1'- and racemic 1,2-disubstituted ferrocenyl phosphine-imidazolium ligands, which are precursors of phosphine-NHCs. 
The precursors for the introduction of the imidazolium moiety are alcohols 3 (Scheme 1) and 6 (Scheme 2). The synthesis of racemic (as well as enantiomerically pure) 1,2-alcohol 6 is already well known. ${ }^{[7,8]}$ We have adapted this method to prepare the new 1,1 '-alcohol 3. It was successfully obtained in two steps from known 1'-diphenylphosphino-1-bromoferrocene, ${ }^{[9]}$ with satisfactory yields.

Procedures for introducing a $N$-substituted imidazole on a ferrocenyl unit can involve the displacement of a chloride, ${ }^{[10]}$ an acetate ${ }^{[6],[11]}$ or a dimethylamino group. ${ }^{[5]}$ Bolm et al. described the efficient conversion of ferrocenyl alcohols into corresponding imidazoliums by a two-step procedure. ${ }^{[12,13]}$ However, this method has not been applied so far to introduce aryl or tertiary alkyl groups. Here the imidazolium functionality has been introduced in one pot from the alcohol, following a protocol developed earlier in our group for the introduction of various nucleophiles on 1,2-disubstituted ferrocenyl phosphines. ${ }^{[14]}$ The $\alpha$-carbocation is generated with a strong acid and reacts with the $N$-R-imidazole ( $\mathbf{a}: \mathrm{R}=\mathrm{Me} ; \mathbf{b} \mathrm{R}=2,4,6-\mathrm{Me}_{3} \mathrm{C}_{6} \mathrm{H}_{2}$ or Mes). This method allowed us to introduce equally well imidazolium groups bearing a primary alkyl or an aryl substituent. The reactions are clean, very rapid and high-yielding (Schemes 1 and 2). ${ }^{[15]}$ The structure of the $4 \mathbf{a}$ and 7a intermediates was confirmed by X-ray diffraction methods (see Fig. 1). The structure of 7a shows two independent molecules in the asymmetric unit, the parameters of which are essentially identical. Only one of them is represented in Fig. 1. The bond lengths and angles in both compounds are all within the expected range. The imidazolium moiety is exo with respect to the ferrocenyl unit, and slightly tilted towards the diphenylphosphino group in the case of 7a. In $\mathbf{4 a}$, the packing is governed by $\mathrm{C}-\mathrm{H}---\pi$ interactions involving the $\mathrm{H}(64)$ of the mesityl group and the centroid of the symmetry-related $\mathrm{Cp}$ ring bearing the mesityl group $\left[\mathrm{C}(64)-\mathrm{H}(64) \ldots \mathrm{Cg} 2^{\mathrm{i}}\right.$ : $\mathrm{C}(64)$ -

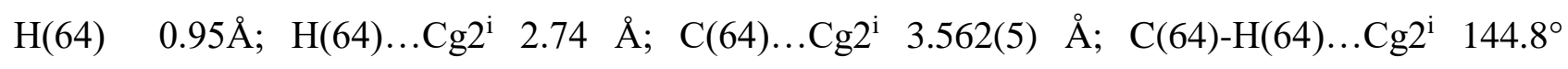
(symmetry code (i): $-x+1,-y+1,-z+1]$, leading to the formation of centrosymmetric pseudo dimer. The two molecules within the asymmetric unit in $\mathbf{7 a}$ are also connected through weak $\mathrm{C}-\mathrm{H}----\pi$ interactions involving the $\mathrm{H}(14)$ atom of the substituted $\mathrm{Cp}$ and the centroid of the $\mathrm{C}(211)-\mathrm{C}(216)$ phenyl ring [C(14)-H(14)...Cg3: C(14)-H(14) $0.93 \AA$; H(14)...Cg3 $3.13 \AA$; C(14)...Cg3 3.755(3) $\AA$; $\left.\mathrm{C}(14)-\mathrm{H}(14) \ldots \mathrm{Cg} 3126.5^{\circ}\right]$. In 7a, the imidazolium and one of the phenyl rings [C(121)-C(126); $\mathrm{C}(221)-\mathrm{C}(226)]$ interact through an offset $\pi-\pi$ stacking with a plane-to-plane distance of 3.566(2)

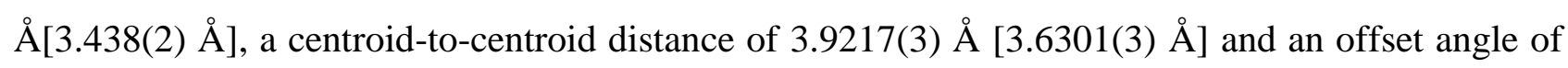
$24.6^{\circ}\left[18.7^{\circ}\right]$. The sulfur atom in $\mathbf{7 a}$ is pointing towards the iron, as has been seen with similar 1,2disubstituted ferrocenyl ligands. ${ }^{[8]}$ Mild reaction conditions were then required for the phosphine deprotection, compatible with the imidazolium moiety. After several attempts, we found that the use of Raney nickel in acetonitrile gave the best results. ${ }^{[16]}$ 
Carbenic rhodium(I) complexes were obtained by deprotonation of the imidazolium salts in the presence of potassium tert-butoxide and $[\mathrm{RhCl}(\mathrm{COD})]_{2}$ in $\mathrm{THF}$, followed by chloride abstraction (Scheme 3). The latter step could be carried out with either $\mathrm{NaBF}_{4}$ in $\mathrm{CH}_{2} \mathrm{Cl}_{2} / \mathrm{H}_{2} \mathrm{O}$, or $\mathrm{AgBF}_{4}$ in $\mathrm{CH}_{2} \mathrm{Cl}_{2}$. The silver reagent results in a faster abstraction process while it does not oxidize the ferrocene unit. Room temperature conditions for the deprotonation step resulted in good yields (75\%) for product $\mathbf{9 a}$, but less satisfactory results were obtained in other cases (i.e. $32 \%$ for $\mathbf{1 0 b}$ ). However, carrying out the deprotonation at $-78^{\circ} \mathrm{C}$ raised the yield of $\mathbf{1 0 b}$ to $77 \%$. This shows that the bulky imidazolium substituent does not negatively affect the coordination process. Good yields $(81 \%)$ were also obtained for $\mathbf{9 b}$ when using the low temperature deprotonation protocol, whereas the yield of 9a was lowered (62\%). Whereas the products with the 1,1'-disubstituted ligands, 9a,b, were obtained selectively, the reactions with the 1,2-disubstituted ligands yielded by-products. The selectivity for 10b was almost total ${ }^{[17]}$, however, complex 10a was obtained along with another carbene-phosphine complex in a $85: 15$ ratio. ${ }^{[18][19]}$ It is reasonable to think that, in the case of 1,2disubstituted ligands, the selectivity is affected by the steric encumbrance of the imidazolium substituent. All complexes are stable in air and could be purified by a filtration on silicagel. The structure of complex 9a was confirmed by X-ray diffraction methods (see Fig. 2). The structure shows a square-planar geometry with the carbene and phosphine donors in a cis arrangment. The bond lengths and angles are again within the expected range for this kind of complexes. The Rhcarbene distance is in accordance with what Seo obtained with a very similar complex, although the Rh-P distance is slightly longer in our case $(2.3345(8) \AA$ instead of $2.2935(10) \AA$ in Seo's Rh(I) complex, which has two NHC-phosphine ligands coordinated to the metal centre). ${ }^{[5]}$ However, we do not observe any significant lenghtening of the Rh-C bonds trans to the carbene (Rh(1)-CG1), with respect to the other $\mathrm{Rh}-\mathrm{C}$ bonds ( $\mathrm{Rh}(1)-\mathrm{CG} 2)$, as is commonly observed for bifunctional NHC ligands. ${ }^{[5,13]}$ Finally, no tilting of the $\mathrm{Cp}$ rings can be observed upon complexation of the ligand to the rhodium centre (the angles between the two least-squares $\mathrm{Cp}$ planes are $4.66(29)^{\circ}$ for $\mathbf{4 a}$ and $3.15(24)^{\circ}$ for $\left.9 \mathbf{a}\right)$.

Preliminary tests have been carried out to evaluate the activity of complexes $\mathbf{9 a}, \mathbf{9 b}$ and $\mathbf{1 0 b}$ (racemic mixture) for the hydrosilylation of ketones. The reaction of diphenylsilane with acetophenone was carried out in dichloromethane or THF at room temperature, with 2 mol\% catalyst (Table 1). The first results show that THF is a better solvent, despite the low solubility of complexes 9a and 9b, and that the complex bearing a 1,2-disubstituted ligand is more active. All complexes show a moderate activity, compared with other literature benchmarks. ${ }^{[13,20]}$

In conclusion, we have prepared new phosphine/imidazolium ferrocenyl ligands by a general and effective method. Further tests will be carried out to evaluate the activity of the $\mathrm{Rh}(\mathrm{I})$ complexes in 
various catalytic reactions. Enantiomerically pure 1,2 ligands will also be prepared and tested in the asymmetric version of the reactions. Results will be published in due course.

\section{Experimental Section}

All reactions were carried out under a dry argon atmosphere using Schlenk glassware and vacuum line techniques. Solvents for syntheses were dried and degassed by standard methods before use. N,N-Dimethylformamide (DMF) was purified by distillation from $\mathrm{CaH}_{2}$. Spectra were recorded on a Brüker AM250, a Brüker AV300 or a Brüker AV500 spectrometer. All spectra were recorded in $\mathrm{CDCl}_{3}$, unless otherwise stated. Mass spectra were obtained from acetonitrile solutions on a TSQ7000 instrument from ThermoElectron. All new compounds described were fully characterised by ${ }^{1} \mathrm{H}$ NMR, ${ }^{13} \mathrm{C}$ NMR, ${ }^{31} \mathrm{P}$ NMR, elemental analysis and mass spectrometry. 1,1'dibromoferrocene and 1'-diphenylphosphino-1-bromoferrocene were prepared according to literature procedures. ${ }^{[9]}$

General Procedures for the Preparation of Imidazolium Salts: To a solution of 3 (100 mg, 0.23 mmol) in degassed dichloromethane $(5 \mathrm{~mL})$ was quickly added $\mathrm{HBF}_{4}\left(35 \mu \mathrm{L}, 54 \%\right.$ wt in $\mathrm{Et}_{2} \mathrm{O}$ ), immediately followed by a $N$-substituted imidazole $(0.35 \mathrm{mmol})$. The mixture was washed with $2 \mathrm{M}$ aq. $\mathrm{HCl}$, water, sat. aq. $\mathrm{NaHCO}_{3}$ and water again. The organic phase was dried $\left(\mathrm{MgSO}_{4}\right)$, filtered and concentrated in vacuo. Methyl-substituted salts : the residue was taken up into dichloromethane $(1 \mathrm{~mL})$, diethyl ether was added and the orange precipitate was filtered, washed with ether and dried in vacuo to give a yellow-orange solid. Mesityl-substituted salts: the residue was purified by column chromatography on silicagel (eluent: $\mathrm{CH}_{2} \mathrm{Cl}_{2}$ /acetone: 9/1) to give a yellow-orange solid. Similar yields were obtained starting from $500 \mathrm{mg}$ of $\mathbf{3}$.

Imidazolium salt 4a: $86 \mathrm{mg}, 64 \%$ yield. Monocrystals suitable for X-ray diffraction studies were obtained by slow evaporation of a dichloromethane solution. $\mathrm{C}_{27} \mathrm{H}_{26} \mathrm{BF}_{4} \mathrm{FeN}_{2} \mathrm{PS}$ (584.21) Calcd: C 55.51, H 4.49, N 4.80\%; found: C 54.98, H 4.16, N 4.55\%; ${ }^{1} \mathrm{H}$ NMR (300 MHz, $\left.\mathrm{CD}_{2} \mathrm{Cl}_{2}, 25{ }^{\circ} \mathrm{C}\right): \delta$ $=8.65\left(\mathrm{~s}, 1 \mathrm{H}, \mathrm{NCHN}{ }^{+}\right), 7.79-7.72\left(\mathrm{~m}, 4 \mathrm{H}, \mathrm{PPh}_{2}\right), 7.59-7.47\left(\mathrm{~m}, 6 \mathrm{H}, \mathrm{PPh}_{2}\right), 7.29\left(\mathrm{~s}, 1 \mathrm{H}, \mathrm{HC}=\mathrm{C} \mathrm{Im}^{+}\right)$, $7.27\left(\mathrm{~s}, 1 \mathrm{H}, \mathrm{HC}=\mathrm{C} \mathrm{Im}{ }^{+}\right), 5.09$ (s, 2H, Cp), 4.69 (s, 2H, Cp), 4.54 (s, 2H, Cp or $\left.\mathrm{CH}_{2} \mathrm{Im}^{+}\right), 4.50$ (s, 2H, Cp or $\left.\mathrm{CH}_{2} \mathrm{Im}^{+}\right), 4.16$ (s, 2H, Cp), $3.88\left(\mathrm{~s}, 3 \mathrm{H}, \mathrm{CH}_{3} \mathrm{Im}^{+}\right) ;{ }^{13} \mathrm{C}\left\{{ }^{1} \mathrm{H}\right\} \mathrm{NMR}\left(75.5 \mathrm{MHz}, \mathrm{CD}_{2} \mathrm{Cl}_{2}, 25\right.$ $\left.{ }^{\circ} \mathrm{C}\right): \delta=135.5\left(\mathrm{NCN}^{+}\right), 134.2\left(\mathrm{~d}, J_{\mathrm{P}, \mathrm{C}}=87.0 \mathrm{~Hz}, 2 \times\right.$ quat $\left.\mathrm{PPh}_{2}\right), 131.55\left(2 \times \mathrm{PPh}_{2}\right), 131.53\left(\mathrm{~d}, J_{\mathrm{P}, \mathrm{C}}\right.$ $\left.\left.\left.=10.6 \mathrm{~Hz}, 4 \times \mathrm{PPh}_{2}\right), 128.4\left(\mathrm{~d}, J_{\mathrm{P}, \mathrm{C}}=12.5 \mathrm{~Hz}, 4 \times \mathrm{PPh}_{2}\right), 123.6(\mathrm{C}=\mathrm{C} \mathrm{Im})^{+}\right), 121.8(\mathrm{C}=\mathrm{C} \mathrm{Im})^{+}\right), 80.4$ (quat $\left.\mathrm{Cp}^{\mathrm{C}}\right), 76.2\left(\mathrm{~d}, J_{\mathrm{P}, \mathrm{C}}=96.9 \mathrm{~Hz}\right.$, quat $\left.\mathrm{Cp}^{\mathrm{P}}\right), 74.1\left(\mathrm{~d}, J_{\mathrm{P}, \mathrm{C}}=12.4 \mathrm{~Hz}, 2 \times \mathrm{Cp}^{\mathrm{P}}\right), 73.1\left(\mathrm{~d}, J_{\mathrm{P}, \mathrm{C}}=10.0\right.$ $\left.\mathrm{Hz}, 2 \times \mathrm{Cp}^{\mathrm{P}}\right), 71.7\left(2 \times \mathrm{Cp}^{\mathrm{C}}\right), 71.3\left(2 \times \mathrm{Cp}^{\mathrm{C}}\right), 49.1\left(C \mathrm{H}_{2} \mathrm{Im}^{+}\right), 36.3\left(C_{3} \mathrm{Im}^{+}\right) ;{ }^{31} \mathrm{P}\left\{{ }^{1} \mathrm{H}\right\} \mathrm{NMR}(81.0$ $\left.\mathrm{MHz}, \mathrm{CD}_{2} \mathrm{Cl}_{2}, 25^{\circ} \mathrm{C}\right): \delta=41.0 ; \mathrm{m} / z(\mathrm{ESI}) 497\left(\mathrm{M}^{+}, 70 \%\right) ; 415\left(\mathrm{M}^{+}-\mathrm{C}_{4} \mathrm{H}_{6} \mathrm{~N}_{2}, 100\right)$.

Imidazolium salt 4b: $96 \mathrm{mg}, 60 \%$ yield. $\mathrm{C}_{35} \mathrm{H}_{34} \mathrm{BF}_{4} \mathrm{FeN}_{2} \mathrm{PS}$ (688.36) Calcd: $\mathrm{C}$ 61.07, $\mathrm{H}$ 4.98, N 4.07\%; found: $\mathrm{C} 60.31, \mathrm{H} 4.71, \mathrm{~N} 3.91 \%$; ${ }^{1} \mathrm{H}$ NMR (300 MHz, $\left.\mathrm{CD}_{3} \mathrm{CN}, 25{ }^{\circ} \mathrm{C}\right): \delta=8.54(\mathrm{~s}, 1 \mathrm{H}$, 
$\left.\mathrm{NCHN}^{+}\right)$, 7.80-7.73 (m, 4H, $\left.\mathrm{PPh}_{2}\right), 7.60-7.50\left(\mathrm{~m}, 7 \mathrm{H}, \mathrm{PPh}_{2}+\mathrm{HC}=\mathrm{C} \mathrm{Im}{ }^{+}\right), 7.40(\mathrm{~s}, 1 \mathrm{H}, \mathrm{HC}=\mathrm{C} \mathrm{Im})^{+}$, 7.11 (s, 2H, Mes), 5.15 (br s, 2H, $\mathrm{CH}_{2} \mathrm{Im}^{+}$), 4.71 (br s, 2H, Cp), 4.55 (br s, 2H, Cp), 4.51 (br s, 2H, $\mathrm{Cp}), 4.18$ (br s, 2H, Cp), 2.36 (s, 3H, p-CH3 Mes), 2.00 (s, 6H, o- $\left.\mathrm{CH}_{3} \mathrm{Mes}\right) ;{ }^{13} \mathrm{C}\left\{{ }^{1} \mathrm{H}\right\} \mathrm{NMR}(75.5$ $\left.\mathrm{MHz}, \mathrm{CD}_{3} \mathrm{CN}, 25{ }^{\circ} \mathrm{C}\right): \delta=141.2$ (quat Mes), $135.8\left(\mathrm{NCN}^{+}\right), 134.7\left(2 \times\right.$ quat Mes), $134.4\left(\mathrm{~d}, J_{\mathrm{P}, \mathrm{C}}=\right.$ 87.0 Hz, $2 \times$ quat $\left.\mathrm{PPh}_{2}\right), 131.7\left(\mathrm{~d}, J_{\mathrm{P}, \mathrm{C}}=2.9 \mathrm{~Hz}, 2 \times \mathrm{PPh}_{2}\right), 131.4\left(\mathrm{~d}, J_{\mathrm{P}, \mathrm{C}}=10.7 \mathrm{~Hz}, 4 \times \mathrm{PPh}_{2}\right)$, 131.0 (quat Mes), $129.5(2 \times \mathrm{C}-\mathrm{H} \mathrm{Mes}), 128.5\left(\mathrm{~d}, J_{\mathrm{P}, \mathrm{C}}=12.5 \mathrm{~Hz}, 4 \times \mathrm{PPh}_{2}\right), 124.2\left(\mathrm{C}=\mathrm{C} \operatorname{Im}^{+}\right)$, $122.7\left(\mathrm{C}=\mathrm{C} \mathrm{Im}^{+}\right), 80.7\left(\right.$ quat $\left.\mathrm{Cp}^{\mathrm{C}}\right), 76.1\left(\mathrm{~d}, J_{\mathrm{P}, \mathrm{C}}=97.3 \mathrm{~Hz}\right.$, quat $\left.\mathrm{Cp}^{\mathrm{P}}\right), 74.0\left(\mathrm{~d}, J_{\mathrm{P}, \mathrm{C}}=12.4 \mathrm{~Hz}, 2 \times\right.$ $\left.\mathrm{Cp}^{\mathrm{P}}\right), 73.2\left(\mathrm{~d}, J_{\mathrm{P}, \mathrm{C}}=10.1 \mathrm{~Hz}, 2 \times \mathrm{Cp}^{\mathrm{P}}\right), 71.6\left(2 \times \mathrm{Cp}^{\mathrm{C}}\right), 71.2\left(2 \times \mathrm{Cp}^{\mathrm{C}}\right), 49.2\left(\mathrm{CH}_{2} \mathrm{Im}^{+}\right), 20.2\left(p-\mathrm{CH}_{3}\right.$ Mes), $16.6\left(2 \times o_{-} \mathrm{CH}_{3} \mathrm{Mes}\right) ;{ }^{31} \mathrm{P}\left\{{ }^{1} \mathrm{H}\right\} \mathrm{NMR}\left(121.5 \mathrm{MHz}, \mathrm{CD}_{3} \mathrm{CN}, 25{ }^{\circ} \mathrm{C}\right): \delta=40.6 ; \mathrm{m} / z(\mathrm{ESI}) 601$ $\left(\mathrm{M}^{+}, 100 \%\right) ; 415\left(\mathrm{M}^{+}-\mathrm{C}_{12} \mathrm{H}_{14} \mathrm{~N}_{2}, 100\right)$.

Imidazolium salt 7a: $96 \mathrm{mg}, 71 \%$ yield. Monocrystals suitable for X-ray diffraction studies were obtained by slow diffusion of diethyl ether into a THF solution. $\mathrm{C}_{27} \mathrm{H}_{26} \mathrm{BF}_{4} \mathrm{FeN}_{2} \mathrm{PS}$ (584.21) Calcd: C 55.51, H 4.49, N 4.80\%; found: C 51.53, H 3.90, N 4.32\%; $\left.{ }^{1} \mathrm{H} \mathrm{NMR} \mathrm{(500} \mathrm{MHz,} \mathrm{CDCl}_{3}, 25{ }^{\circ} \mathrm{C}\right): \delta$ $=8.33\left(\mathrm{~s}, 1 \mathrm{H}, \mathrm{NCHN}^{+}\right), 7.73-7.33\left(\mathrm{~m}, 10 \mathrm{H}, \mathrm{PPh}_{2}\right), 6.98\left(\right.$ br s, $\left.1 \mathrm{H}, \mathrm{HC}=\mathrm{C} \mathrm{Im}{ }^{+}\right), 6.87($ br s, $1 \mathrm{H}$, $\left.\mathrm{HC}=\mathrm{C} \mathrm{Im}{ }^{+}\right), 6.41\left(\right.$ br s, $\left.1 \mathrm{H}, \mathrm{CH}_{2} \mathrm{Im}^{+}\right), 5.15-5.04\left(\mathrm{~m}, 2 \mathrm{H}, \mathrm{CH}_{2} \mathrm{Im}^{+}+\mathrm{Cp}\right), 4.52$ (br s, 1H, Cp), 4.41 (br s, 5H, Cp'), 3.81 (br s, $1 \mathrm{H}, \mathrm{Cp}$ ), 3.52 (br s, 3H, CH3 $\left.\mathrm{Im}^{+}\right) ;{ }^{13} \mathrm{C}\left\{{ }^{1} \mathrm{H}\right\} \mathrm{NMR}\left(125.8 \mathrm{MHz}, \mathrm{CDCl}_{3}, 25\right.$ $\left.{ }^{\circ} \mathrm{C}\right): \delta=135.9\left(\mathrm{NCN}^{+}\right), 134.0\left(\mathrm{~d}, J_{\mathrm{P}, \mathrm{C}}=85.6 \mathrm{~Hz}, 2 \times\right.$ quat $\left.\mathrm{PPh}_{2}\right), 132.2-131.8\left(\mathrm{~m}, 5 \times \mathrm{PPh}_{2}\right), 131.4$ $\left(\mathrm{d}, J_{\mathrm{P}, \mathrm{C}}=10.1 \mathrm{~Hz}, 2 \times \mathrm{PPh}_{2}\right), 128.7\left(\mathrm{~d}, J_{\mathrm{P}, \mathrm{C}}=12.6 \mathrm{~Hz}, \mathrm{PPh}_{2}\right), 128.45\left(\mathrm{~d}, J_{\mathrm{P}, \mathrm{C}}=12.6 \mathrm{~Hz}, 2 \times \mathrm{PPh}_{2}\right)$, $123.6\left(\mathrm{C}=\mathrm{C}, \mathrm{Im}^{+}\right), 122.4\left(\mathrm{C}=\mathrm{C}, \mathrm{Im}^{+}\right), 83.4\left(\mathrm{~d}, J_{\mathrm{P}, \mathrm{C}}=11.3 \mathrm{~Hz}\right.$, quat $\left.\mathrm{Cp}\right), 77.5-77.0\left(\mathrm{Cp}+\mathrm{CDCl}_{3}\right)$, $76.3\left(\mathrm{~d}, J_{\mathrm{P}, \mathrm{C}}=11.3 \mathrm{~Hz}, \mathrm{Cp}\right), 74.3\left(\mathrm{~d}, J_{\mathrm{P}, \mathrm{C}}=94.4 \mathrm{~Hz}\right.$, quat Cp), $71.4\left(\mathrm{Cp}^{\prime}\right), 71.3(\mathrm{Cp}), 48.2\left(\mathrm{CH}_{2} \mathrm{Im}^{+}\right)$, $37.0\left(\mathrm{CH}_{3} \mathrm{Im}^{+}\right) ;{ }^{31} \mathrm{P}\left\{{ }^{1} \mathrm{H}\right\} \mathrm{NMR}\left(202.5 \mathrm{MHz}, \mathrm{CDCl}_{3}, 25{ }^{\circ} \mathrm{C}\right): \delta=40.7 ; \mathrm{m} / z(\mathrm{ESI}) 497\left(\mathrm{M}^{+}, 37 \%\right)$; $415\left(\mathrm{M}^{+}-\mathrm{C}_{4} \mathrm{H}_{6} \mathrm{~N}_{2}, 100\right)$.

Imidazolium salt 7b: $121 \mathrm{mg}, 76 \%$ yield. $\mathrm{C}_{35} \mathrm{H}_{34} \mathrm{BF}_{4} \mathrm{FeN}_{2} \mathrm{PS}$ (688.36) Calcd: $\mathrm{C} 61.07, \mathrm{H} 4.98, \mathrm{~N}$ 4.07\%; found: C 60.34, H 4.93, N 3.98\%; ${ }^{1} \mathrm{H}$ NMR (300 MHz, $\left.\mathrm{CDCl}_{3}, 25{ }^{\circ} \mathrm{C}\right): \delta=8.67(\mathrm{~s}, 1 \mathrm{H}$, $\left.\mathrm{NCHN}^{+}\right)$, 7.84-7.77 (m, 2H, PPh $)$, 7.58-7.28 (m, 9H, PPh $\left.2+\mathrm{HC}=\mathrm{C} \mathrm{Im}{ }^{+}\right), 6.92(\mathrm{~s}, 2 \mathrm{H}, \mathrm{Mes}), 6.71$ $\left.(\mathrm{s}, 1 \mathrm{H}, \mathrm{HC}=\mathrm{C} \mathrm{Im})^{+}\right), 6.66\left(\mathrm{~d}, J_{\mathrm{H}, \mathrm{H}}=14.3 \mathrm{~Hz}, 1 \mathrm{H}, \mathrm{CH}_{2} \mathrm{Im}^{+}\right), 5.58\left(\mathrm{~d}, J_{\mathrm{H}, \mathrm{H}}=14.3 \mathrm{~Hz}, 1 \mathrm{H}, \mathrm{CH}_{2} \mathrm{Im}^{+}\right)$, 5.31 (br s, 1H, Cp), 4.56 (br s, 1H, Cp), 4.35 (s, 5H, Cp'), 3.96 (br s, 1H, Cp), 2.30 (s, 3H, p-CH Mes), 1.84 (br s, $3 \mathrm{H}, o-\mathrm{CH}_{3} \mathrm{Mes}$ ), 1.68 (br s, 3H, o- $\left.\mathrm{CH}_{3} \mathrm{Mes}\right) ;{ }^{13} \mathrm{C}\left\{{ }^{1} \mathrm{H}\right\} \mathrm{NMR}\left(75.5 \mathrm{MHz}, \mathrm{CDCl}_{3}\right.$, $\left.25^{\circ} \mathrm{C}\right): \delta=141.2$ (quat Mes), $136.2\left(\mathrm{NCN}^{+}\right), 135.0\left(\mathrm{~d}, J_{\mathrm{P}, \mathrm{C}}=86.0 \mathrm{~Hz}\right.$, quat $\left.\mathrm{PPh}_{2}\right), 134.2$ (quat Mes), $132.3\left(\mathrm{~d}, J_{\mathrm{P}, \mathrm{C}}=87.3 \mathrm{~Hz}\right.$, quat $\left.\mathrm{PPh}_{2}\right), 132.0\left(\mathrm{~d}, J_{\mathrm{P}, \mathrm{C}}=11.0 \mathrm{~Hz}, 2 \times \mathrm{PPh}_{2}+\mathrm{d}, J_{\mathrm{P}, \mathrm{C}}=3.1 \mathrm{~Hz}, \mathrm{PPh}_{2}\right)$, $131.7\left(\mathrm{~d}, J_{\mathrm{P}, \mathrm{C}}=2.9 \mathrm{~Hz}, \mathrm{PPh}_{2}\right), 131.4\left(\mathrm{~d}, J_{\mathrm{P}, \mathrm{C}}=10.5 \mathrm{~Hz}, 2 \times \mathrm{PPh}_{2}\right), 130.5($ quat Mes), $129.7(\mathrm{C}-\mathrm{H}$ Mes), $128.6\left(\mathrm{~d}, J_{\mathrm{P}, \mathrm{C}}=12.4 \mathrm{~Hz}, 2 \times \mathrm{PPh}_{2}\right), 128.3\left(\mathrm{~d}, J_{\mathrm{P}, \mathrm{C}}=12.7 \mathrm{~Hz}, 2 \times \mathrm{PPh}_{2}\right), 122.9\left(\mathrm{C}=\mathrm{C} \mathrm{Im}^{+}\right)$, $122.2\left(\mathrm{C}=\mathrm{C} \mathrm{Im}{ }^{+}\right), 83.6\left(\mathrm{~d}, J_{\mathrm{P}, \mathrm{C}}=12.3 \mathrm{~Hz}\right.$, quat $\left.\mathrm{Cp}^{\mathrm{C}}\right), 76.9\left(\mathrm{~d}, J_{\mathrm{P}, \mathrm{C}}=8.6 \mathrm{~Hz}, \mathrm{Cp}\right), 75.7\left(\mathrm{~d}, J_{\mathrm{P}, \mathrm{C}}=11.4\right.$ $\mathrm{Hz}, \mathrm{Cp}), 72.9\left(\mathrm{~d}, J_{\mathrm{P}, \mathrm{C}}=94.5 \mathrm{~Hz}\right.$, quat $\left.\mathrm{Cp}^{\mathrm{P}}\right), 71.8\left(\mathrm{~d}, J_{\mathrm{P}, \mathrm{C}}=10.1 \mathrm{~Hz}, \mathrm{Cp}\right), 71.4\left(\mathrm{Cp}^{\prime}\right), 48.0\left(C_{2} \mathrm{Im}^{+}\right)$, 
$21.0\left(p-\mathrm{CH}_{3} \mathrm{Mes}\right), 17.2\left(2 \times o-\mathrm{CH}_{3} \mathrm{Mes}\right) ;{ }^{31} \mathrm{P}\left\{{ }^{1} \mathrm{H}\right\} \mathrm{NMR}\left(121.5 \mathrm{MHz}, \mathrm{CDCl}_{3}, 25{ }^{\circ} \mathrm{C}\right): \delta=40.4$; $m / z(\mathrm{ESI}) 601\left(\mathrm{M}^{+}, 100 \%\right) ; 415\left(\mathrm{M}^{+}-\mathrm{C}_{12} \mathrm{H}_{14} \mathrm{~N}_{2}, 27\right) ; 187$ (20).

Suppoprting Information (see footnote on the first page of this article): full experimental details,

${ }^{1} \mathrm{H}-,{ }^{13} \mathrm{C}$ - and ${ }^{31} \mathrm{P}$ NMR data, mass spectral data and elemental analysis for all new compounds. Crystal data for $4 \mathbf{a}, 7 \mathbf{a}$ and $9 \mathbf{a}$.

CCDC 616649-616651 contain the supplementary crystallographic data for this paper. These data can be obtained free of charge from The Cambridge Crystallographic Data Centre via www.ccdc.cam.ac.uk/datarequest/cif.

\section{Acknowledgements}

We thank the CNRS for support of this work and Yannick Coppel for NMR analysis of the rhodium complexes.

\section{References}

[1] D. Bourissou, O. Guerret, F. P. Gabbai, G. Bertrand, Chem. Rev. 2000, 100, 39.

[2] W. A. Herrmann, Angew. Chem. Int. Ed. 2002, 41, 1290-1309; N. M. Scott, S. P. Nolan, Eur. J. Inorg. Chem. 2005, 1815-1828.

[3] N. Stylianides, A. A. Danopoulos, N. Tsoureas, J. Organomet. Chem. 2005, 690, 59485958; L. D. Field, B. A. Messerle, K. Q. Vuong, P. Turner, Organometallics 2005, 24, 4241-4250.

[4] C. Yang, H. M. Lee, S. P. Nolan, Org. Lett. 2001, 3, 1511-1514; J. Wolf, A. Labande, J.-C. Daran, R. Poli, J. Organomet. Chem. 2006, 691, 433-443; J. Wolf, A. Labande, M. Natella, J.-C. Daran, R. Poli, J. Mol. Cat. A 2006, 259, 205-212.

[5] H. Seo, H.-J. Park, B. Y. Kim, J. H. Lee, S. U. Son, Y. K. Chung, Organometallics 2003, 22, 618-620.

[6] S. Gischig, A. Togni, Organometallics 2004, 23, 2479-2487.

[7] T. Hayashi, T. Mise, M. Fukushima, M. Kagotani, N. Nagashima, Y. Hamada, A. Matsumoto, S. Kawakami, M. Konishi, K. Yamamoto, M. Kumada, Bull. Chem. Soc. Jpn. 1980, 53, 1138-1151.

[8] N. Mateus, L. Routaboul, J.-C. Daran, E. Manoury, J. Organomet. Chem. 2006, 691, 22972310.

[9] T.-Y. Dong, L.-L. Lai, J. Organomet. Chem. 1996, 509, 131-134; T.-Y. Dong, P.-H. Ho, C.K. Chang, J. Chin. Chem. Soc. 2000, 47, 421-424.

[10] K. S. Coleman, S. Turberville, S. I. Pascu, M. L. H. Green, J. Organomet. Chem. 2005, 690, 653-658.

[11] H. Seo, B. Y. Kim, J. H. Lee, H.-J. Park, S. U. Son, Y. K. Chung, Organometallics 2003, 22, 4783-4791.

[12] C. Bolm, M. Kesselgruber, G. Raabe, Organometallics 2002, 21, 707-710.

[13] Y. Yuan, G. Raabe, C. Bolm, J. Organomet. Chem. 2005, 690, 5747-5752.

[14] L. Routaboul, S. Vincendeau, J.-C. Daran, E. Manoury, Tetrahedron: Asymmetry 2005, 16, 2685-2690.

[15] Any attempt to prepare the imidazolium salts by other methods (generation of chloride, bromide or acetate leaving groups from alcohol $\mathbf{3}$ failed to give the expected compound, or the reactions were nor selective neither effective. 
[16] D. Liu, W. Tang, X. Zhang, Org. Lett. 2004, 6, 513-516.

[17] Along with the doublet attributed to $10 \mathrm{~b}$ in ${ }^{31} \mathrm{P}$ NMR, a second, weak doublet can be observed. However, no other compound can be detected apart from $\mathbf{1 0 b}$ in the ${ }^{1} \mathrm{H}$ NMR spectrum.

[18] The yield of 10a was not given, as we obtained an inseparable mixture of two compounds.

[19] The ${ }^{1} \mathrm{H}$ NMR signals of the minor compound indicate that it might be a rhodium complex bearing two carbene-phosphine ligands and no COD.

[20] V. César, S. Bellemin-Laponnaz, H. Wadepohl, L. H. Gade, Chem. Eur. J. 2005, 11, 28622873. 
Table 1 Hydrosilylation of acetophenone with diphenylsilane. Conds: 1 eq. acetophenone, 1.1 eq. diphenylsilane, $2 \mathrm{~mol} \%$ catalyst, room temperature.

\begin{tabular}{ccccc}
\hline Entry & Complex & Solvent & $\mathrm{t}$ (days) & Conversion $(\%)^{\mathrm{a}}$ \\
\hline 1 & $\mathbf{9 a}$ & $\mathrm{CH}_{2} \mathrm{Cl}_{2}$ & 7 & 17 \\
2 & $\mathbf{9 a}$ & $\mathrm{THF}$ & 3 & 46 \\
3 & $\mathbf{9 b}$ & $\mathrm{THF}$ & 3 & 49 \\
4 & $\mathbf{1 0 b}$ & $\mathrm{THF}$ & 3 & 67 \\
\hline${ }^{\mathrm{a}}$ Determined by ${ }^{1} \mathrm{H}$ NMR after hydrolysis of the silylated intermediate.
\end{tabular}

\section{Schemes and figures}

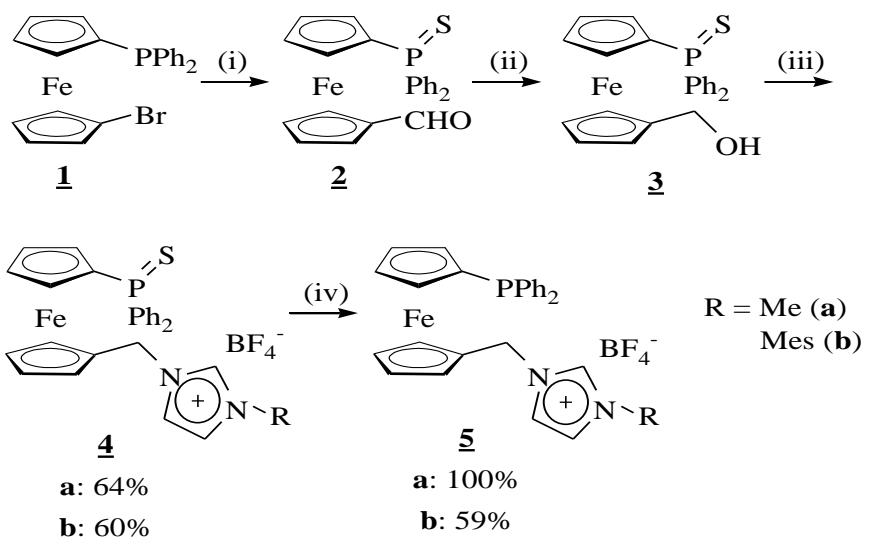

Scheme 1. Synthesis of $1,1^{\prime}$-ferrocenyl ligands.

i. a) $n$-BuLi, THF, $-25^{\circ} \mathrm{C}$, b) $\mathrm{DMF},-25^{\circ} \mathrm{C}$, c) $\mathrm{S}_{8}, \mathrm{CH}_{2} \mathrm{Cl}_{2}, 40^{\circ} \mathrm{C}(70 \%)$; ii. $\mathrm{NaBH}_{4}$, toluene/ $\mathrm{NaOH}$, $0^{\circ} \mathrm{C}(88 \%)$; iii. a) $\mathrm{HBF}_{4}, \mathrm{CH}_{2} \mathrm{Cl}_{2}, \mathrm{rt}$, b) $\mathrm{N}$-R imidazole; iv) Raney $\mathrm{Ni}, \mathrm{MeCN}$, rt or $80^{\circ} \mathrm{C}$.

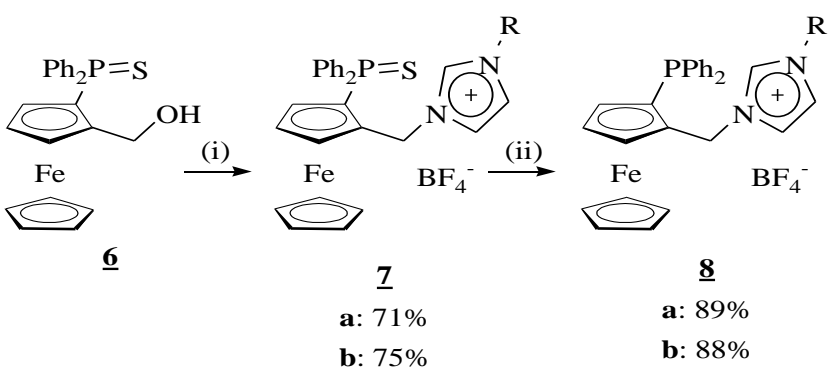

Scheme 2 Synthesis of 1,2-ferrocenyl ligands.

i. a) $\mathrm{HBF}_{4}, \mathrm{CH}_{2} \mathrm{Cl}_{2}$, rt, b) $\mathrm{N}$-R imidazole; ii) Raney $\mathrm{Ni}, \mathrm{MeCN}$, rt or $80^{\circ} \mathrm{C}$. 

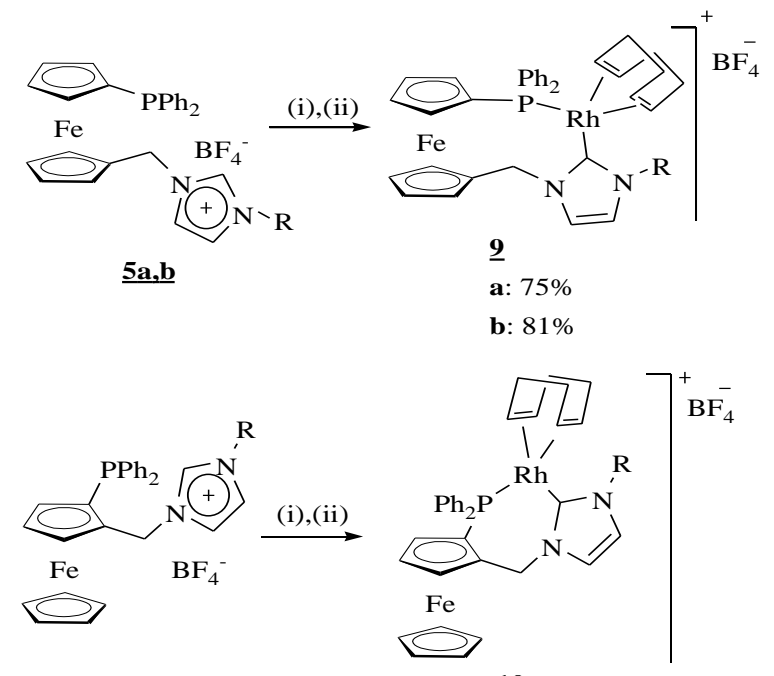

$\underline{\mathbf{8}, \mathbf{b}}$

$\underline{10}$

a (impure)

b: $77 \%$

Scheme 3. Synthesis of Rh complexes

i. $t$-BuOK, $[\mathrm{Rh}(\mathrm{COD}) \mathrm{Cl}]_{2}, \mathrm{THF}$; ii. $\mathrm{NaBF}_{4}, \mathrm{CH}_{2} \mathrm{Cl}_{2} / \mathrm{H}_{2} \mathrm{O}$, rt or $\mathrm{AgBF}_{4}, \mathrm{CH}_{2} \mathrm{Cl}_{2}$, rt.
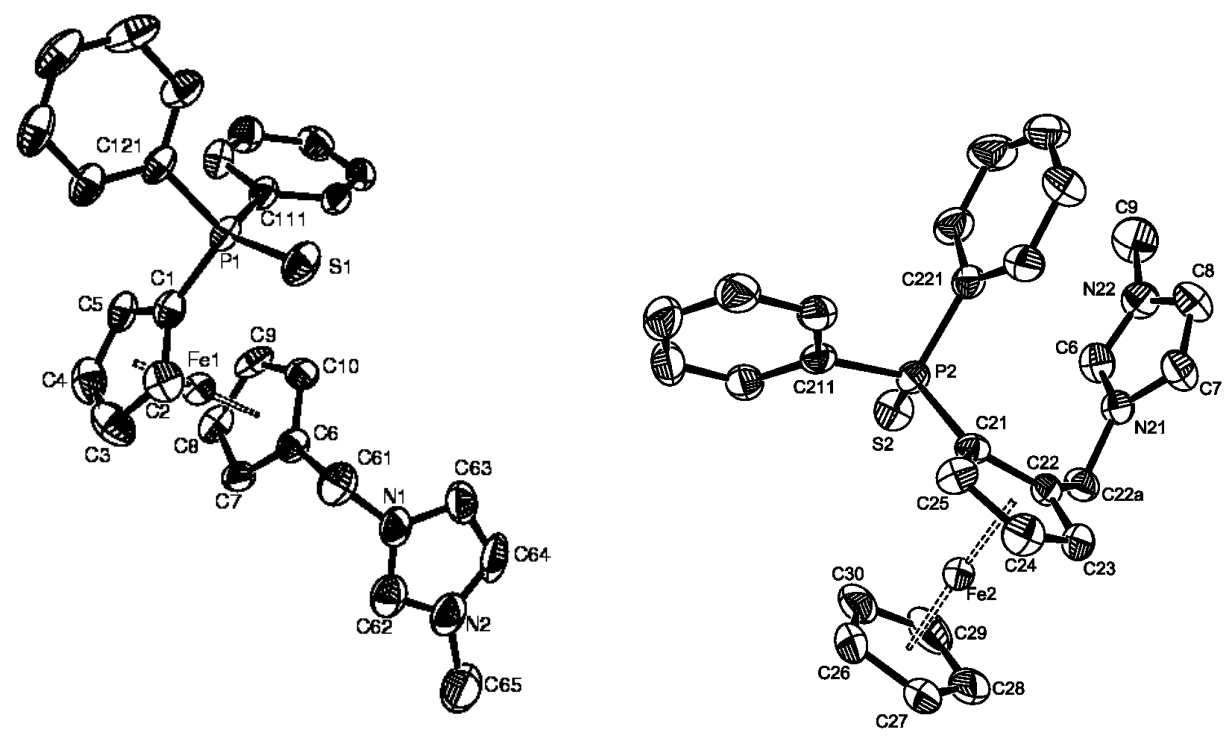

Figure 1 Molecular views of compounds 4a (left) and 7a (right) with atom labelling scheme. Ellipsoids are plotted at the $50 \%$ level. $\mathrm{H}$ atoms have been omitted for clarity. Selected bond lengths $(\AA)$ and bond angles $\left({ }^{\circ}\right), 4 a: C(1)-\mathrm{P}(1)$ 1.790(3), C(62)-N(1) 1.324(5), C(62)-N(2) 1.313(5), C(63)$\mathrm{C}(64)$ 1.330(6); N(2)-C(62)-N(1) 108.6(3); 7a: C(21)-P(2) 1.801(3), C(6)-N(21) 1.316(3), C(6)$\mathrm{N}(22) 1.323(3), \mathrm{C}(7)-\mathrm{C}(8)$ 1.344(4); $\mathrm{N}(21)-\mathrm{C}(6)-\mathrm{N}(22)$ 109.4(3). 


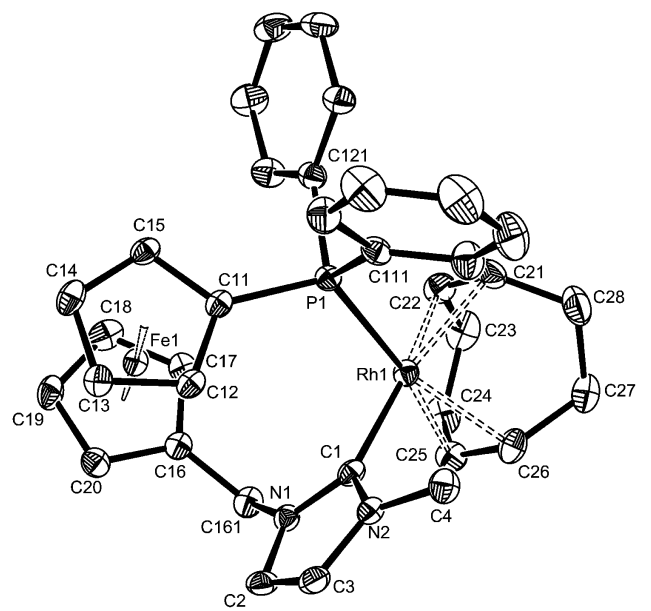

Figure 2 Molecular view of compound 9a with atom labelling scheme. Ellipsoids are plotted at the $50 \%$ level. $\mathrm{H}$ atoms have been omitted for clarity. Selected bond lengths $(\AA)$ and bond angles $\left({ }^{\circ}\right)$ : $\mathrm{Rh}(1)-\mathrm{C}(1)$ 2.047(3), $\mathrm{Rh}(1)-\mathrm{P}(1)$ 2.3345(8), Rh(1)-C(21) 2.215(3), $\mathrm{Rh}(1)-\mathrm{C}(22)$ 2.231(3), $\mathrm{Rh}(1)-$ $\mathrm{C}(25)$ 2.220(3), Rh(1)-C(26) 2.217(3), Rh(1)-CG1 2.1140(3), Rh(1)-CG2 2.1091(3), N(1)-C(1) 1.359(4), N(2)-C(1) 1.357(4), C(2)-C(3) 1.338(4); C(1)-Rh(1)-P(1) 90.83(8), C(1)-Rh(1)-CG1 175.91(8), C(1)-Rh(1)-CG2 90.46(8), CG1-Rh(1)-P(1) 93.26(2), CG2-Rh(1)-P(1) 174.69(2), CG2$\mathrm{Rh}(1)-\mathrm{CG} 1$ 85.450(10), N(1)-C(2)-N(2) 104.5(2). CG1 and CG2 denote respectively the C(21)$\mathrm{C}(22)$ centroïd and the $\mathrm{C}(25)-\mathrm{C}(26)$ centroïd. 


\section{Graphical abstract}

New, bifunctional 1,1'- and 1,2-phosphine-NHC ferrocenyl ligands have been accessed by a general and simple method from the corresponding 1,1'- and 1,2-thiophosphine-alcohols and used to prepare $\mathrm{Rh}^{\mathrm{I}}$ complexes.

Keywords: Carbene ligands / Phosphane ligands / Ferrocenes / Rhodium

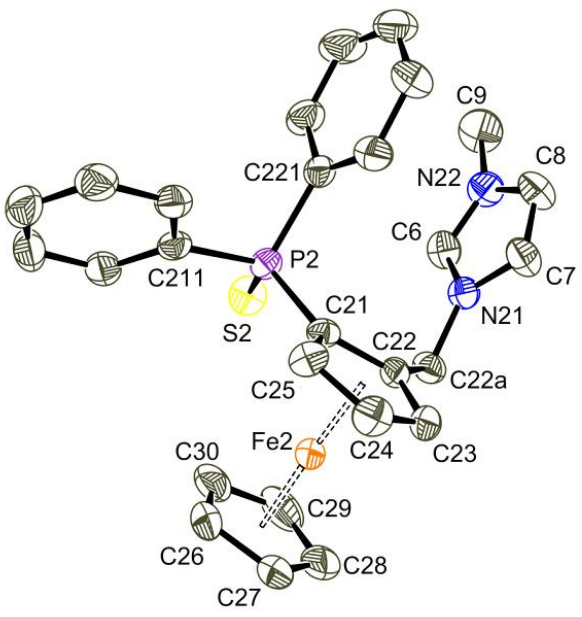

